\title{
A CHARACTERIZATION OF MINIMAL HOMOGENEOUS BANACH SPACES
}

\author{
HANS G. FEICHTINGER
}

\begin{abstract}
Let $G$ be a locally compact group. It is shown that for a homogeneous Banach space $B$ on $G$ satisfying a slight additional condition there exists a minimal space $B_{\min }$ in the family of all homogeneous Banach spaces which contain all elements of $B$ with compact support. Two characterizations of $B_{\min }$ are given, the first one in terms of "atomic" representations. The equivalence of these two characterizations is derived by means of certain (bounded) partitions of unity which are of interest for themselves.
\end{abstract}

Notations. In the sequel $G$ denotes a locally compact group. $|M|$ denotes the (Haar) measure of a measurable subset $M \subseteq G$, or the cardinality of a finite set. $\Re(G)$ denotes the space of continuous, complex-valued functions on $G$ with compact support (supp). For $y \in G$ the (left) translation operator $L_{y}$ is given by $L_{y} f(x)=f\left(y^{-1} x\right)$. A translation invariant Banach space $B$ is called a homogeneous Banach space (in the sense of Katznelson [9]) if it is continuously embedded into the topological vector space $L_{\text {loc }}^{1}(G)$ of all locally integrable functions on $G$, satisfies $\left\|L_{y} f\right\|_{B}=\|f\|_{B}$ for all $y \in G$, and $\lim _{y \rightarrow e}\left\|L_{y} f-f\right\|_{B}=0$ for all $f \in B$ (hence, as usual, two measurable functions coinciding l.a.e. are identified). If, furthermore, $B$ is a dense subspace of $L^{1}(G)$ it is called a Segal algebra (in the sense of Reiter [15], [16]). Any homogeneous Banach space is a left $L^{1}(G)$-Banachmodule with respect to convolution, i.e. $f \in B, h \in L^{1}(G)$ implies $h * f \in B$, and $\|h * f\|_{B}<\|h\|_{1}\|f\|_{B}$; in particular any Segal algebra is a Banach ideal of $L^{1}(G)$. In the sequel we shall write $B_{\Omega}$ for the space $\{f \mid f \in B$, supp $f$ compact $\}$.

LEMMA 1. Let $V=V^{-1}$ be an open, relatively compact subset of $G$. Then there exists a subset $Y=\left(y_{i}\right)_{i \in I} \subseteq G$ such that

$$
G=\bigcup_{i \in I} y_{i} V^{2}
$$

and

$$
\sup _{y \in G}\left|\left\{i \mid y K_{1} \cap y_{i} K_{2} \neq \varnothing\right\}\right|<\left|K_{1} K_{2}^{-1} V\right||V|^{-1}<\infty
$$

for all pairs $K_{1}, K_{2}$ of compact sets in $G$. In particular, $\left(y_{i} W\right)_{i \in I}$ defines a locally finite covering of $G$ for any compact subset $W \supseteq V^{2}$.

Received by the editors December 17, 1979.

1980 Mathematics Subject Classification. Primary 43A15, 22D15.

Key words and phrases. Homogeneous Banach spaces, Segal algebras, Besov spaces, Banach modules. 
Proof. By Zorn's lemma there exists for each $V$ a set $Y=\left\{y_{i}\right\}_{i \in I}$ that is maximal among those sets $Z$ such that

$$
z_{1} V \cap z_{2} V=\varnothing \text { for } z_{1}, z_{2} \in Z, z_{1} \neq z_{2} \text {. }
$$

Then $G=\cup_{i \in I} y_{i} V^{2}$, and (3) implies for any pair $K_{1}, K_{2}$ of compact sets and $y \in G$

$$
\left|\left\{i \mid y K_{1} \cap y_{i} K_{2} \neq \varnothing\right\}\right| \leqslant\left|\left\{i \mid y K_{1} K_{2}^{-1} V \supset y_{i} V\right\}\right|<\left|K_{1} K_{2}^{-1} V\right||V|^{-1} .
$$

RemarK 1. This result coincides with Lemma 3.2 of [2]. It is related to the covering lemma due to Emerson and Greanleaf ([4], cf. also [13], or [1, p. 70]).

THEOREM 2 (PARTITION OF UNITY). Let $B$ be a homogeneous Banach space on $G$ that contains positive functions $u \neq 0$ with arbitrary small support, and let $W$ be an open subset of $G$. Then there exists a bounded partition of unity $\left(\varphi_{i}\right)_{i \in I} \subseteq B$, i.e.,

$$
\sum_{i \in I} \varphi_{i}(x) \equiv 1 \text { and } \sup _{i \in I}\left\|\varphi_{i}\right\|_{B}<C<\infty
$$

and

$$
\text { supp } \varphi_{i} \subseteq y_{i} W \text { for all } i \in I \text {. }
$$

Furthermore, the family $\left(y_{i}\right)_{i \in I}$ satisfies (2) for some $V$ where $V^{4} \subseteq W$.

Proof. Choose $V$ and $\left\{y_{i}\right\}$ such that $V=V^{-1}, V^{4} \subseteq W$ and such that $\left\{y_{i}\right\}$ satisfies Lemma 1. Then whenever $\psi \in \Re^{+}(G)$ has $\psi=1$ on $V^{2}$, supp $\psi \subseteq V^{3}$ and $0 \leqslant \psi \leqslant 1$, the function $\Psi$ defined by $\Psi=\Sigma_{i \in I} L_{y} \psi$ satisfies

$$
1<\Psi(x)<C_{K} \text { for all } x \in G,
$$

where $C_{K}$ is independent of $\psi$. If we set $\psi_{i}=\left(L_{y_{i}} \psi\right) \Psi^{-1}$ we have

$$
\sum_{i \in I} \psi_{i}(x) \equiv 1, \quad \operatorname{supp} \psi_{i} \subseteq y_{i} V^{3}, \quad 0<\psi_{i}(x)<1 \text { for } x \in G .
$$

Choose now $u \in B$ such that $u>0,\|u\|_{1}=1$, supp $u \subseteq V$. Then we have $\left(\Sigma \psi_{i}\right) * u=\Sigma\left(\psi_{i} * u\right) \equiv 1$. Set $\varphi_{i}=\psi_{i} * u$. Then

$$
\begin{aligned}
& \sum \varphi_{i}(x) \equiv 1, \quad \operatorname{supp} \varphi_{i} \subseteq y_{i} V^{4} \subseteq y_{i} W, \quad \text { and } \\
& \sup _{i \in I}\left\|\varphi_{i}\right\|_{B} \leqslant \sup _{i \in I}\left\|\psi_{i}\right\|_{1}\|u\|_{B}<\left|V^{3}\right|\|u\|_{B}<\infty .
\end{aligned}
$$

General hypothesis. From now on we suppose the following situation to be given:

$B$ is a homogeneous Banach space on $G$, which is a Banach module (with respect to pointwise multiplication) over a homogeneous function algebra $A$, i.e., we suppose that $A \subseteq C^{b}(G)$ is a homogeneous Banach space as well as a regular, selfadjoint Banach algebra (with pointwise multiplication), such that $\|h f\|_{B}<$ $\|h\|_{A}\|f\|_{B}$ for all $h \in A, f \in B$.

REMARK 2. For many examples of spaces $B$ there exists a natural Banach algebra $A^{1} \subseteq L^{\infty}(G)$ satisfying only $L^{1} * A^{1} \subseteq A^{1}$ and $\left\|L_{y} h\right\|_{A^{1}}=\|h\|_{A^{1}}$ for all $h \in A^{1}$, $y \in G\left(\right.$ e.g. $\left.A^{1}=L^{\infty}(G)\right)$. Then, however

$$
A_{c}^{1}:=\left\{h \mid h \in A^{1},\left\|L_{y} h-h\right\|_{A^{1}} \rightarrow 0 \text { for } y \rightarrow e\right\}
$$


is a homogeneous function algebra, i.e. the general hypothesis is satisfied for $A=A_{c}^{1}$.

Now we can give a first characterization of the minimal homogeneous Banach space containing all elements of $B$ with compact support.

Theorem 3. Let $B$ satisfy the general hypothesis. Then there exists a minimal space $B_{\min }$ in the family of all homogeneous Banach spaces $C$ satisfying $B_{\boldsymbol{R}} \subseteq C$. $B_{\min }$ can be characterized as follows: Let an open, relatively compact set $Q \subseteq G$ be given. We set

$$
\begin{aligned}
B_{\min }=\left\{f \mid f=\sum_{n} L_{y_{n}} f_{n}, y_{n} \in G, f_{n}\right. & \in B, \\
& \left.\quad \operatorname{supp} f_{n} \subseteq Q \text { for } n>1, \sum_{n}\left\|f_{n}\right\|_{B}<\infty\right\},
\end{aligned}
$$

and

$$
\|f\|_{\min }=\inf \left\{\sum\left\|f_{n}\right\|_{B}, f=\sum L_{y_{n}} f_{n}\right\},
$$

the infimum in (11) being taken over the representations of $f$ as in (10). $B \cap \AA(G)$ is a dense subspace of $B_{\min }$, and if $B$ contains positive elements with arbitrary small support, then $B_{\min }$ is a Segal algebra.

Proof. It is a matter of routine to verify that \|\|$_{\min }$ defines a translation invariant, complete norm on $B_{\text {min }}$. Furthermore, it follows from the definition that the elements of the form $g=\sum_{n=1}^{k} L_{y_{n}} f_{n}$ constitute a dense subspace of $B_{\min }$. It will therefore be sufficient to show that $\left\|L_{z} g-g\right\|_{\min } \rightarrow 0$ for $z \rightarrow e$ for such $g$ 's. We have

$$
\begin{aligned}
\left\|L_{z} g-g\right\|_{\min } & =\left\|\sum_{n=1}^{k} L_{y_{n}}\left(L_{y_{n}^{-1} z y_{n}} f_{n}-f_{n}\right)\right\|_{\min } \\
& <\sum_{n=1}^{k}\left\|L_{y_{n}^{-1} z y_{n}} f_{n}-f_{n}\right\|_{B}<\varepsilon,
\end{aligned}
$$

if we choose $U \subseteq G$ such that $\left\|L_{y_{n}^{-1} z y_{n}} f_{n}-f_{n}\right\|_{B}<\varepsilon / k$ for all $z \in U$ and $\left(y_{n}^{-1} U y_{n}\right) \operatorname{supp} f_{n} \subseteq Q, 1 \leqslant n \leqslant k$. (It is now clear that $B_{\min }$ is a homogeneous Banach space continuously embedded in $B$. Also, $g$ can be approximated by elements of the form $u * g \in\left(\Re^{+}(G) * L_{\Omega}^{1}\right) \cap B \subseteq \Re(G) \cap B$.)

Let now $f \in B_{\Omega}$ be given. Then (by Theorem 2) there exists a finite set $F \subseteq I$ such that $\Sigma_{i \in F} \varphi_{i}(x)=1$ for all $x \in \operatorname{supp} f$, and $\operatorname{supp} \varphi_{i} \subseteq y_{i} Q$. That implies

$$
f=\sum_{i \in F} \varphi_{i} f=\sum_{i \in F} L_{y_{i}}\left(L_{y_{i}^{-1}}\left(\varphi_{i} f\right)\right) \text {. }
$$

Since $\operatorname{supp}\left(L_{y_{i}^{-1}}\left(\varphi_{i} f\right)\right) \subseteq y_{i}^{-1} \operatorname{supp} \varphi_{i} \subseteq Q$ for all $i \in F, f$ belongs to $B_{\min }$ as a consequence of the general hypothesis.

Let now $C$ be any homogeneous Banach space such that $B_{\Omega} \subseteq C$. Then, by the closed graph theorem, there exists $C_{1}>0$ such that $\|f\|_{C}<C_{1}\|f\|_{B}$ for all $f \in B$ satisfying supp $f \subseteq Q^{\sim}$. That implies that, for all $f \in B_{\min }$,

$$
\|f\|_{C}<\sum_{n}\left\|L_{y_{n}} f_{n}\right\|_{C}=\sum_{n}\left\|f_{n}\right\|_{C}<C_{1} \sum_{n}\left\|f_{n}\right\|_{B}
$$


for any representation of $f$. Hence, $B_{\min } \subseteq C$, and $\|f\|_{C}<C_{1}\|f\|_{\min }$ for all $f \in$ $B_{\min }$. The proof is now complete.

It should be mentioned that the above characterization also implies that different relatively compact sets $Q$ with nonvoid interior define the same space with an equivalent norm. That can also be shown directly, using Theorem 2 (for similar assertions cf. [1], [2], [7] or [12]).

Corollary 4. Let $A$ be homogeneous function algebra. Then $A_{\min }$ is a Segal algebra on $G$. In particular, $A_{\min }$ is a Banach ideal in $L^{1}(G)$ (with convolution) as well as in $A$ (pointwise multiplication). Furthermore, $A_{\min }$ is the minimal element in the family of all homogeneous Banach spaces which are at the same time Banach-Amodules with respect to pointwise multiplication.

Proof. Since $A$ is regular and selfadjoint, there exists elements $u \in A, u>0$, with arbitrary small (compact) support (contained in $V=V^{-1}$ ). Hence, $A_{\min }$ is a Segal algebra by Theorem 3 . Since, for obvious reasons, $A_{\min }$ is also a (pointwise) Banach- $A$-module, it is sufficient to show that any homogeneous space $C$ with that property satisfies $C \supseteq A_{\Omega}$. Let $f \in A_{\Omega}$, with supp $f=K \subseteq G$ be given, and let $u$ be as above. Choose now any $k \in \mathfrak{\Omega}^{+}(G)$ such that $k(x)=1$ for all $x \in K V$. Then $k * u(z)=1$ for all $z \in K$, and $k * u \in \Re(G) * A \subseteq A$. We obtain $f=(k * u) f \in$ $A B \subseteq B$, and our assertion is verified.

Next we make use of the partition of unity in order to give another description of $B_{\text {min }}$

TheORem 5. Let $B$ satisfy the general hypothesis, and let $g \in A_{\Re}, g \neq 0$ be given such that $g(x)=$ const on some open set $U \subseteq G$. Then

$$
B_{\min }=B_{1}=\left\{f \in B_{\text {loc }},\|f\|_{B_{1}}=\int_{G}\left\|\left(L_{,} g\right) f\right\|_{B} d y<\infty\right\},
$$

and the norms \|\|$_{B_{1}}$ and \|\|$_{\min }$ are equivalent on $B_{\min }$.

Proof. It follows from (10) that we have $B_{\min } \subseteq B_{1}$, provided that there exists $C_{1}>0$ such that

$$
\sup _{z \in G}\left\|L_{z} f\right\|_{B_{1}}<C_{1}\|f\|_{B} \text { for all } f \in B \text {, with supp } f \subseteq Q .
$$

But (13) follows from this:

$$
\int_{G}\left\|L_{y} g L_{z} f\right\|_{B} d y<|z(\operatorname{supp} f)(\operatorname{supp} g)|\|g\|_{A}\|f\|_{B}
$$

There remains the difficult part of the proof, i.e. the inclusion $B_{1} \subseteq B_{\min }$. The following lemma will be used.

LemMa 6. Let $\left(\varphi_{i}\right)_{i \in I} \subseteq A$ be a partition of unity, as in Theorem 2, bounded in $A$. Then there exists $C_{2}>0$ such that for any subset $J \subseteq I$ the function $\varphi_{J}=\Sigma_{i \in J} \varphi_{i}$ satisfies

$$
\left\|f \varphi_{J}\right\|_{B_{1}}<C_{2}\|f\|_{B_{1}} \text { for all } f \in B_{1} \text {. }
$$


Proof. Making use of (2) we have, for all $f \in B_{1}$

$$
\left\|f \varphi_{J}\right\|_{B_{1}}=\int_{G}\left\|L_{y} g\left(f \varphi_{J}\right)\right\|_{B} d y<\int_{G} \sum\left\|L_{y} g\left(f \varphi_{i}\right)\right\|_{B} d y
$$

(the sum being taken over all $i$ such that $\left.y(\operatorname{supp} g) \cap \operatorname{supp} \varphi_{i} \neq \varnothing\right)$

$$
<\sup _{i \in I}\left\|\varphi_{i}\right\|_{A} \cdot\left|(\operatorname{supp} g) V^{5}\right||V|^{-1} \int_{G}\left\|\left(L_{y} g\right) f\right\|_{B} d y=C_{2}\|f\|_{B_{1}} .
$$

Proof of Theorem 5 (CONTINUEd). Suppose now there exists $f \in B_{1} \backslash B_{\min }$. Since $B_{1}$ is a translation invariant space, we may suppose that $U$ is a neighborhood of the identity and $g(x)=1$ on $U$. Let $V=V^{-1}$ be an open set such that $V^{5} \subseteq U$, and let $\psi \in A$ be such that

$$
\psi(x) \equiv 1 \text { for all } x \in V^{5} \text {, and supp } \psi \subseteq V^{6} .
$$

Let further $\left(\varphi_{i}\right)_{i \in I}$ be the bounded partition of unity in $A$ given by Theorem 2 (with $\left.W=V^{4}\right)$. Then, by Lemma 6

$$
\int_{G}\left\|(L, \psi) f \varphi_{J}\right\|_{B} d y<\|\psi\|_{A} C_{2} \int_{G}\left\|\left(L_{y} g\right) f\right\|_{B} d y<\infty
$$

for all subsets $J \subseteq I$. On the other hand $f \notin B_{\min }$ implies that for any $k \in \mathbf{N}$ there exists $I(k)=\left\{y_{1}, \ldots, y_{s(k)}\right\} \subseteq I$ such that

$$
\sum_{s=1}^{s(k)}\left\|f \varphi_{s}\right\|_{B}>k,
$$

$f=\sum_{i} L_{y_{i}}\left(L_{y_{i}-1} f \varphi_{i}\right)$ being a representation of $g$ as in (10) (recall that $\operatorname{supp}\left(f \varphi_{i}\right) \subseteq$ $y_{i} V^{4}$; cf. (8)). We may suppose $\left\|f \varphi_{s}\right\|_{B}>\left\|f \varphi_{s+1}\right\|_{B}$ for all $s$. Now we claim that for some $C_{3}>0$ it is possible to choose a subset $J(k)=\left\{y_{s_{1}}, \ldots, y_{s_{m(k)}}\right\}$ such that

$$
y_{s_{n}} V^{7} \cap y_{s_{m}} V^{4} \neq \varnothing \text { for } n \neq m \text {, and } s_{n}<C_{3} n \text {. }
$$

That subsequence can be obtained by induction. Given $s_{1}, \ldots, s_{r}$ one sets

$$
s_{r+1}=\min \left[s \mid\left\|f \varphi_{s}\right\|_{B}=\max \left\{\left\|f \varphi_{n}\right\|_{B}, y_{n} \notin \bigcup_{i=1}^{r} y_{s_{i}} V^{11}\right\}\right] .
$$

Since for $i$ fixed, there are at most $\left|V^{12}\right||V|^{-1}\left(=C_{3}\right)$ distinct elements in $\left(y_{s}\right)_{s=1}^{s(k)}$ (cf. (2)) such that $y_{s} \in y_{s_{i}} V^{11}$, we obtain $s_{r+1}<C_{3} r+1<C_{3}(r+1)$. Fix now $s_{i} \in J(k)$. Then, by (18), for $y \in y_{s_{i}} V$,

$$
\operatorname{supp}(L, \psi) \cap y_{s_{j}} V^{4} \subseteq y_{s_{i}} V^{7} \cap y_{s, j} V^{4}=\varnothing \quad \text { when } j \neq i .
$$

On the other hand (15) and (8) imply

$$
L_{y} \psi(x)=1 \text { for } x \in y_{i} V^{4} \supset \operatorname{supp} \varphi_{i} .
$$

It follows from (20) and (21) that one has

$$
\left\|L_{y} \psi\left(f \varphi_{J(k)}\right)\right\|_{B}=\left\|L_{y} \psi\left(f \varphi_{i}\right)\right\|_{B}=\left\|f \varphi_{i}\right\|_{B} \quad \text { for } y \in y_{i} V, i \in J(k) .
$$

That and (16) yield

$$
\begin{aligned}
\sum_{i \in J(k)}\left\|f \varphi_{i}\right\|_{B} & <|V|^{-1} \sum_{i \in J(k)} \int_{y_{i} V}\left\|L_{y} \psi\left(f \varphi_{J(k)}\right)\right\|_{B} d y \\
& <\left(|V|^{-1}\|g\|_{A} C_{2}\right)\|f\|_{B_{1}}
\end{aligned}
$$


However, (23) gives a contradiction to (17). That completes the proof.

REMARK 3. It is not difficult to show that one can drop the condition $g=$ const on $U$ if $A$ possesses "local inverses", i.e. if $A$ is a standard function algebra as considered in [15, Chapter II] (e.g. $A=C^{0}(G)$, or $A=A(G)=\left\{\hat{f} \mid f \in L^{1}(G)\right\}$, the Fourier algebra). In particular, any two nonzero elements $g_{1}, g_{2} \in A_{\Omega}$ define equivalent norms on $B_{\min }$. Going back to the characterization of $B_{\min }$ given in Corollary 4 one can even show that $f \in A_{\min }$ iff $\int_{G}\left\|f\left(L_{y} f\right)\right\|_{A} d y<\infty$ in this case.

REMARK 4. It follows from the proof of Theorem 5 that $\Sigma_{i \in I}\left\|f \varphi_{i}\right\|_{B}$ defines another equivalent norm on $B_{\min }$ for any bounded partition of unity $\left(\varphi_{i}\right)_{i \in I} \subseteq A_{1}$ as considered in Theorem 2 .

Applications. For $A=C^{0}(G)$ (space of continuous functions on $G$ vanishing at infinity) Theorem 5 gives the main result of [6] (cf. [1, Proposition VIII], and [12]). We have called $\left(C^{0}(G)\right)_{\min }$ "Wiener's algebra" because it coincides with Wiener's classical algebra for $G=\mathbf{R}^{n}$. For $G$ Abelian, Theorem 5 coincides with the main result of [14] that has been proved there using structure theory. As already observed in [14] that allows the characterization of certain algebras of functions considered in [11] by a minimality property. For $A=A(G)$ (Fourier algebra) the same result has been proved already in [7, II] (Theorem 1.1), also using structure theory. It can be shown that the space $(A(G))_{\min }$ has a number of interesting properties which suggest it might become a useful tool in Harmonic Analysis. It will be treated in detail in a subsequent paper.

The choice $B=L^{p}(G), 1<p<\infty\left(A=C^{0}(G)\right)$ gives a family of Segal algebras, defined for arbitrary locally compact groups. Using essentially the arguments applied in the proof of Theorem 5 one shows that $\left(L^{p}\right)_{\min }$ coincides with $l^{1}\left(L^{p}\right)(G$ Abelian, cf. [1]), with $W^{p}$ (see [10]), or $\left(L^{p}, l^{1}\right)\left(G=\mathbf{R}^{n}\right.$, cf. [7]), or with $L_{p, 1}$ as defined in [2]. In particular, all these spaces coincide for an arbitrary locally compact group (as far as they are well defined). It is left to the reader to check the details.

It is clear that more general spaces are obtained if one replaces the scale of $L^{p}$-spaces by a more general family of solid Banach function spaces, e.g. Lorentz spaces or Orlicz spaces. The main advantage of the approach given above relies on the fact that our characterization is not limited to the case of solid spaces. It is also possible to consider $B_{\min }$ for homogeneous Banach spaces $B$ whose elements satisfy certain smoothness conditions. Thus, for example, our results apply to the classical Lipschitz spaces $\operatorname{lip} \alpha, 0<\alpha<1$, since these spaces are homogeneous function algebras on $\mathbf{R}^{n}$ (cf. [19, 2.8]). These spaces, in turn, may be considered as the most simple examples (corresponding to $p=q=\infty$ ) of Besov spaces. The most general family of examples in this direction are the so-called spaces of Besov-Hardy-Sobolev-Triebel type $B_{p, q}^{s}$ and $F_{p, q}^{s}$ on $\mathbf{R}^{n}$, with $1 \leqslant p, q<\infty, s>0$. These two three-parameter families of spaces are treated in detail by Triebel in [17] and [18]. According to Theorem 2.6.1 of [18] these spaces satisfy the general hypothesis for $A^{1}=C^{r}, r \geqslant r(p, q, s): C^{r}=\left\{f \mid D^{\alpha} f \in C^{b}\left(\mathbf{R}^{m}\right)\right.$ for $\left.|\alpha| \leqslant r\right\}$, with the norm $\|f\|_{C^{\prime}}$ $=\Sigma_{|\alpha|<m}\left\|D^{\alpha} f\right\|_{\infty}\left(\right.$ cf. Remark 2), or for $A=$ closure of $C_{\Re}^{\infty}\left(\mathbf{R}^{n}\right)$ in $C^{r}$ (for a related statement see [3, Theorem 4.5]). 
We conclude by mentioning that $(A(G))_{\min }$ is also well defined for arbitrary locally compact groups, if one takes $A(G)$ to Eymard's Fourier algebra in this more general context. This, in turn, may be considered as the special $p=2$ of the $A^{p}(G)$-algebras of C. Herz (cf. [5]).

ACKnowledgement. The author is indebted to J. P. Bertrandias for discussions on related topics during a visit in Grenoble. At this occasion he also pointed out the utility of bounded partitions of unity to the author.

Added in proof. A summary of results concerning $(A(G))_{\min }=: S_{0}(G)$ and its dual (treated in [7]) are given in the paper Un espace de Banach de distributions tempérées sur les groupes localement compacts abéliens, C. R. Acad. Sci. Paris 290 (1980), 791-794. Generalizations of the spaces considered above and in [1], [2], [6]-[8], [10] and [12] are to be treated in forthcoming papers.

\section{REFERENCES}

1. J. P. Bertrandias, C. Datry and C. Dupuis, Unions et intersections $L^{p}$ invariantes par translation ou convolution, Ann. Inst. Fourier (Grenoble) 28 (1978), 58-84.

2. R. C. Busby and H. A. Smith, Product-convolution operators and mixed-norm spaces, Trans. Amer. Math. Soc. (to appear).

3. W. C. Connett and A. L. Schwartz, The theory of ultraspherical multipliers, Mem. Amer. Math. Soc. No. 183 (1977).

4. W. R. Emerson and F. P. Greanleaf, Covering properties and Foelner conditions for locally compact groups, Math. Z. 102 (1967), 370-384.

5. P. Eymard, Algèbres $A_{p}$ et convoluteurs de $L^{p}$, Séminaire Bourbaki No. 367, Novembre 1969.

6. H. G. Feichtinger, A characterization of Wiener's algebra on locally compact groups, Arch. Math. (Basel) 29 (1977), 136-140.

7. __ The minimal strongly character invariant Segal algebra. I, II (preprints, Wien 1978/79).

8. F. Holland, Harmonic analysis on amalgams of $L^{p}$ and $l^{q}$, J. London Math. Soc. (2) 10 (1975), 295-305.

9. Y. Katznelson, An introduction to harmonic analysis, Wiley, New York, 1968.

10. H. E. Krogstad, Multipliers of Segal algebras, Math. Scand. 38 (1976), 285-303.

11. H. Leptin, On onesided harmonic analysis in non commutative locally compact groups, J. Reine Angew. Math. 306 (1979), 122-153.

12. T. S. Liu, A. van Rooij and J. K. Wang, On some group algebra modules related to Wiener's algebra $M_{1}$, Pacific J. Math. 55 (1974), 507-520.

13. P. Milnes and J. V. Bondar, A simple proof of a covering property of locally compact groups, Proc. Amer. Math. Soc. 73 (1979), 117-118.

14. D. Poguntke, Gewisse Segalsche Algebren auf lokalkompakten Gruppen, Arch. Math. Basel 33 (1980), 454-460.

15. H. Reiter, Classical harmonic analysis and locally compact groups, Oxford Univ. Press, London, 1968.

16. __ , L $L^{1}$-algebras and Segal algebras, Lecture Notes in Math., Vol. 231, Springer-Verlag, Berlin and New York, 1971.

17. H. Triebel, Interpolation theory, function spaces, differential operators, North-Holland, Amsterdam, 1978.

18. __ Spaces of Besov-Hardy-Sobolev type, Teubner, Leipzig, 1978.

19. H. C. Wang, Homogeneous Banach algebras, Lecture Notes in Pure and Appl. Math., vol. 29, Marcel Dekker, New York, 1977.

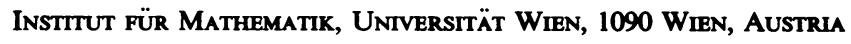

